\title{
Issues for a mathematical definition of LES*
}

\author{
Jean-Luc Guermond ${ }^{1}$ and Serge Prudhomme ${ }^{2}$ \\ 1 Texas A\&M University, College Station TX 77843, USA, and LIMSI, CNRS \\ UPR 3251, BP 133 Orsay Cedex, France, guermond@math.tamu.edu \\ 2 ICES, The University of Texas at Austin, TX 78712, USA, \\ serge@ices.utexas.edu
}

Summary. The mathematical foundations of Large Eddy Simulation (LES) for three-dimensional turbulent incompressible viscous flows are discussed and the notion of suitable approximations is introduced.

\section{Introduction}

\subsection{What is LES?}

Since the early work of [7], Large Eddy Simulation (LES) has become over the years an increasingly popular method, as evidenced by the vast amount of publications on the subject in the literature, and is now considered a tool of choice for simulating three-dimensional incompressible viscous flows at large Reynolds numbers. Heuristically speaking, Large Eddy models are obtained by applying a low-pass filter to the Navier-Stokes equations. The filtered equations are then similar to the original equations but for the presence of the so-called subgrid scale stresses accounting for the influence of the small scales onto the large ones. Assuming that the behavior of the small scales is almost universal, the objective of LES is to model the subgrid scale stresses (the so-called closure problem) and to compute the dynamics of the large scales by using the filtered equations. Although this description of LES is widely accepted, it nevertheless falls short of an unambiguous mathematical theory. Our impression is that LES is at the present time a fuzzy concept. Some authors think of LES as the solution to the filtered equations whereas others think of it as finite-dimensional approximations thereof. Others expect LES to reproduce the statistics of the large scales instead of approximating

\footnotetext{
* This paper has been published in Numerical Mathematics and Advanced Applications, Proceedings of ENUMATH 2005, 6th European Conference on Numerical Mathematics and Advanced Applications, A. Berúdez de Castro, D. Gómez, P. Quintela, P. Salgado (Eds.), Santiago de Compostela, Spain, July 2005, SpringerVerlag Berlin Heidelberg (2006) 796-804.
} 
individual solutions. It is also common practice to invoke the filtering of length scales without defining the filter being used or to outright ignore the concept of filter when modeling the subgrid scale tensor. Another common unjustified practice consists of assuming that the filtering length scale is equal to the mesh size of the approximation method that is used, regardless on the method in question.

In an attempt to address some of the above issues, we are currently developing a research program aiming at constructing a framework for a mathematical theory of LES. The present paper makes a first step is this direction by introducing the concept of suitable approximation (see $\S 2.1$ ). We show that the construction of suitable approximations shares many heuristic features with what is often referred to in the engineering literature as LES modeling. The proposal made in this paper is that the notion of suitable approximations be a concept that, together with other mathematical criteria yet to be clearly identified, should be seriously considered as part of any future mathematical definition of LES.

\subsection{Suitable weak solutions}

Let $\Omega \subset \mathbb{R}^{3}$ be an open smooth, bounded, connected domain occupied by a viscous fluid. Let $(0, T)$ be a time interval. It is generally accepted that the Navier-Stokes equations accurately model the behavior of turbulent incompressible flows of the fluid in $\Omega$ :

$$
\left\{\begin{array}{l}
\partial_{t} \mathbf{u}+\mathbf{u} \cdot \nabla \mathbf{u}+\nabla p-\nu \nabla^{2} \mathbf{u}=\mathbf{f} \quad \text { in } Q_{T}, \\
\nabla \cdot \mathbf{u}=0 \quad \text { in } Q_{T},\left.\quad \mathbf{u}\right|_{\Gamma}=0 \text { or } \mathbf{u} \text { is periodic, }\left.\quad \mathbf{u}\right|_{t=0}=u_{0},
\end{array}\right.
$$

where $\mathbf{u}$ and $p$ are the velocity and the pressure respectively, $Q_{T}=\Omega \times(0, T)$, $\Gamma$ is the boundary of $\Omega, \mathbf{u}_{0}$ the solenoidal initial data, $\mathbf{f}$ a source term, $\nu$ the viscosity, and the density is chosen equal to unity. The problem is nondimensionalized, i.e., $\nu$ is the inverse of the Reynolds number.

To implicitly account for boundary conditions, we introduce

$$
\begin{aligned}
& \mathbf{X}= \begin{cases}\mathbf{H}_{0}^{1}(\Omega) & \text { If Dirichlet conditions, } \\
\mathbf{H}_{\#}^{1}(\Omega)=\left\{\mathbf{v} \in \mathbf{H}^{1}(\Omega), \mathbf{v} \text { periodic }\right\} & \text { If periodic conditions. }\end{cases} \\
& \mathbf{V}=\{\mathbf{v} \in \mathbf{X}, \nabla \cdot \mathbf{v}=0\}, \quad \mathbf{H}=\overline{\mathbf{V}}^{\mathbf{L}^{2}}
\end{aligned}
$$

Henceforth we focus our interest on suitable weak solutions to (1), [10].

Definition 1. A weak solution to the Navier-Stokes equation $(\mathbf{u}, p)$ is suitable if $\mathbf{u} \in L^{2}(0, T ; \mathbf{X}) \cap L^{\infty}\left(0, T ; \mathbf{L}^{2}(\Omega)\right), p \in L^{\frac{5}{4}}\left(Q_{T}\right)$ and the local energy balance

$$
\partial_{t}\left(\frac{1}{2} \mathbf{u}^{2}\right)+\nabla \cdot\left(\left(\frac{1}{2} \mathbf{u}^{2}+p\right) \mathbf{u}\right)-\nu \nabla^{2}\left(\frac{1}{2} \mathbf{u}^{2}\right)+\nu(\nabla \mathbf{u})^{2}-\mathbf{f} \cdot \mathbf{u} \leq 0
$$

is satisfied in the distributional sense in $Q_{T}$. 
To the present time, the best partial regularity result available for (1) is the so-called Caffarelli-Kohn-Nirenberg Theorem [1] proving that the onedimensional Hausdorff measure of the set of singularities of a suitable weak solution is zero. By analogy with nonlinear conservative laws, (4) can be viewed as an entropy-like condition which may (hopefully?) selects the physical solutions of (1). Whether suitable weak solutions are indeed classical is not known. Moreover, despite the fact that the result of the CKN Theorem also holds for weak solutions[6], it is not known whether weak solutions are in fact suitable.

\section{2 suitable approximations}

\subsection{Suitable approximations}

A general definition for LES is out of the scope of the present paper, but we believe that a reasonable definition should at least be founded on the following criteria: (1) A LES approximation should be finite-dimensional, i.e., it should be computable; (2) A LES approximation should solve a problem which is consistent with the Navier-Stokes equations; (3) A sequence of LES approximations should select a physical solution of the Navier-Stokes equations under the appropriate limiting process, i.e., one which is suitable.

We collect the above three criteria by defining the notion of suitable approximation as follows:

Definition 2. A sequence $\left(\mathbf{u}_{\gamma}, p_{\gamma}\right)_{\gamma>0}$ with $\mathbf{u}_{\gamma} \in L^{\infty}\left(0, T ; \mathbf{L}^{2}(\Omega)\right) \cap L^{2}(0, T ; \mathbf{X})$ and $p_{\gamma} \in \mathcal{D}^{\prime}\left((0, T), L^{2}(\Omega)\right)$ is said to be a suitable approximation to (1) if

i. There are two finite-dimensional vectors spaces $\mathbf{X}_{\gamma} \subset \mathbf{X}$ and $M_{\gamma} \subset L^{2}(\Omega)$ such that $\mathbf{u}_{\gamma} \in \mathcal{C}^{0}\left([0, T] ; \mathbf{X}_{\gamma}\right)$ and $p_{\gamma} \in L^{2}\left((0, T) ; M_{\gamma}\right)$ for all $T>0$.

ii. The sequence converges (up to subsequences) to a weak solution of (1), say $\mathbf{u}_{\gamma} \rightarrow \mathbf{u}$ weakly in $L^{2}(0, T ; \mathbf{X})$ and $p_{\gamma} \rightarrow p$ in $\mathcal{D}^{\prime}\left((0, T), L^{2}(\Omega)\right)$.

iii. The weak solution $(\mathbf{u}, p)$ is suitable.

\subsection{Practical construction of suitable approximations}

In practice, the construction of a suitable approximations can be decomposed into the following three steps:

(1) Construction of what we hereafter call the pre-LES-model. This step consists of regularizing the Navier-Stokes equations by introducing a regularization parameter $\varepsilon$ associated with some filtering of the Navier-Stokes equations. This parameter is a user-defined length scale of the smallest eddies that are allowed to be nonlinearly active in the flow. The purpose of the regularization technique is to yield a well-posed problem for all times. Moreover, the limit solution of the pre-LES-model must be a weak solution to the Navier-Stokes equations as $\varepsilon \rightarrow 0$ and should be suitable. The pre-LES-model can be thought of as a filtered version of the Navier-Stokes equations where 
the subgrid scale stresses have been modeled in such a way that the resulting PDE is well-posed and yields a unique weak solution that converges (up to subsequences) to a suitable weak solution to the Navier-Stokes equations.

(2) Discretization of the pre-LES-model. This step introduces the meshsize parameter $h$ associated with the size of the smallest scale that can be represented in the finite-dimensional spaces $\mathbf{X}_{\gamma}, M_{\gamma}$; roughly $\operatorname{dim}\left(\mathbf{X}_{\gamma}\right)=$ $\mathcal{O}\left((L / h)^{3}\right)$ where $L=\operatorname{diam}(\Omega)$.

(3) Determination of a (possibly maximal) relationship between $\varepsilon$ and $h$. The large eddy scale $\varepsilon$ and the mesh size $h$ must be selected in such a way that the sequence of discrete solutions is ensured to converge to a suitable solution of the Navier-Stokes equations when $\varepsilon \rightarrow 0$ and $h \rightarrow 0$. In the above definition the parameter $\gamma$ is a yet to be specified combination of the two parameters $h$ and $\varepsilon$ that reminds us that the process $\lim _{\varepsilon \rightarrow 0, h \rightarrow 0}$ is a distinguished limit.

\section{Review of existing pre-LES-models}

We show in this section that some of the regularization techniques recognized in the literature as LES models are indeed pre-LES-models in the sense of our definition, i.e., they all select suitable solutions as $\varepsilon \rightarrow 0$.

\subsection{Hyperviscosity}

Lions [9] proposed the following hyperviscosity model:

$$
\left\{\begin{array}{l}
\partial_{t} \mathbf{u}_{\varepsilon}+\mathbf{u}_{\varepsilon} \cdot \nabla \mathbf{u}_{\varepsilon}+\nabla p_{\varepsilon}-\nu \nabla^{2} \mathbf{u}_{\varepsilon}+\varepsilon^{2 \alpha}\left(-\nabla^{2}\right)^{\alpha} \mathbf{u}_{\varepsilon}=\mathbf{f} \quad \text { in } Q_{T}, \\
\nabla \cdot \mathbf{u}_{\varepsilon}=0 \quad \text { in } Q_{T}, \\
\left.\mathbf{u}_{\varepsilon}\right|_{\Gamma}, \ldots,\left.\partial_{n}^{\alpha-1} \mathbf{u}_{\varepsilon}\right|_{\Gamma}=0, \quad \text { or } \mathbf{u}_{\varepsilon} \text { is periodic }\left.\quad \mathbf{u}\right|_{t=0}=\mathbf{u}_{0}
\end{array}\right.
$$

where $\varepsilon>0$ and $\alpha$ is an integer. Hyperviscosity models are frequently used in so-called LES simulations of oceanic and atmospheric flows or to control the Navier-Stokes equations. The appealing aspects of this regularization are that it yields a well-posed problem in the classical sense when $\alpha \geq \frac{5}{4}$ in three space dimensions and that limit solutions as $\varepsilon \rightarrow 0$ are suitable.

\subsection{Leray mollification}

A simple construction yielding suitable solutions has indeed been proposed by Leray [8] before this very notion was introduced in the literature.

Assume that $\Omega$ is the three-dimensional torus $(0,2 \pi)^{3}$ and let $\left(\phi_{\varepsilon}\right)_{\varepsilon>0}$ be a sequence of non-negative mollifying functions. Leray suggested to regularize the Navier-Stokes equations as follows:

$$
\left\{\begin{array}{l}
\partial_{t} \mathbf{u}_{\varepsilon}+\left(\phi_{\varepsilon} * \mathbf{u}_{\varepsilon}\right) \cdot \nabla \mathbf{u}_{\varepsilon}+\nabla p_{\varepsilon}-\nu \nabla^{2} \mathbf{u}_{\varepsilon}=\phi_{\varepsilon} * \mathbf{f}, \\
\nabla \cdot \mathbf{u}_{\varepsilon}=0, \quad \mathbf{u}_{\varepsilon} \text { is periodic, }\left.\quad \mathbf{u}_{\varepsilon}\right|_{t=0}=\phi_{\varepsilon} * \mathbf{u}_{0} .
\end{array}\right.
$$


The mollification device has been introduced by Leray to prove the existence of weak solutions to (1). Quite amazingly not only the pair $\left(\mathbf{u}_{\varepsilon}, p_{\varepsilon}\right)$ converges to a weak solution to (1), but the weak solution in question is also suitable. Roughly speaking, the convolution process removes scales that are smaller than $\varepsilon$. Hence, by using $\phi_{\varepsilon} * \mathbf{u}_{\varepsilon}$ as the advection velocity, scales smaller than $\varepsilon$ are not allowed to be nonlinearly active. This is a feature shared by most LES models.

\subsection{Leray- $\alpha$ model}

A variant of the Leray mollification consists of the so-called Leray- $\alpha$ model

$$
\left\{\begin{array}{l}
\partial_{t} \mathbf{u}_{\varepsilon}+\overline{\mathbf{u}_{\varepsilon}} \cdot \nabla \mathbf{u}_{\varepsilon}-\nu \nabla^{2} \mathbf{u}_{\varepsilon}+\nabla \pi_{\varepsilon}=\mathbf{f},\left.\quad \mathbf{u}_{\varepsilon}\right|_{\Gamma}=0 \text { or } \mathbf{u}_{\varepsilon} \text { is periodic } \\
\left(I-\varepsilon^{2} \nabla^{2}\right) \overline{\mathbf{u}_{\varepsilon}}=\mathbf{u}_{\varepsilon},\left.\quad \overline{\mathbf{u}_{\varepsilon}}\right|_{\Gamma}=0 \text { or } \overline{\mathbf{u}_{\varepsilon}} \text { is periodic } \\
\nabla \cdot \overline{\mathbf{u}_{\varepsilon}}=0,\left.\quad \mathbf{u}_{\varepsilon}\right|_{t=0}=\mathbf{u}_{0}
\end{array}\right.
$$

as introduced in [2]. Once again, regularization yields existence and uniqueness in the large. Moreover, when periodic boundary conditions are enforced the pair $\left(\mathbf{u}_{\varepsilon}, p_{\varepsilon}\right)$ converges, up to subsequences, to a suitable solution.

\subsection{Nonlinear Galerkin Method (NLGM)}

We focus in this section on the Nonlinear Galerkin Method as introduced in [3]. Let $\Omega$ be the torus $(0,2 \pi)^{3}$. Let $\mathbb{P}_{N}$ be the set of trigonometric polynomials of partial degree at most $N: \mathbb{P}_{N}=\left\{p(\mathbf{x})=\sum_{|\mathbf{k}|_{\infty} \leq N} c_{\mathbf{k}} e^{i \mathbf{k} \cdot \mathbf{x}}, c_{\mathbf{k}}=\bar{c}_{-\mathbf{k}}\right\}$, and denote by $\dot{\mathbb{P}}_{N}$ the subspace of $\mathbb{P}_{N}$ composed of the trigonometric polynomials of zero mean value. For any $\mathbf{k} \in \mathbb{Z}$, we denote by $|\mathbf{k}|$ the Euclidean norm of $\mathbf{k}$ and by $|\mathbf{k}|_{\infty}$ the maximum norm. We denote by $\bar{z}$ the conjugate of $z$. Let $\varepsilon>0$ be a large eddy scale. Let us set $N=\frac{1}{\varepsilon}$ (or the integer the closest to $\left.\frac{1}{\varepsilon}\right)$. We now introduce the following finite-dimensional vector spaces:

$$
\mathbf{X}_{\varepsilon}=\dot{\mathbb{P}}_{N}, \quad \text { and } \quad M_{\varepsilon}=\dot{\mathbb{P}}_{N},
$$

Let $P_{\varepsilon}: \mathbf{H}_{\#}^{1}(\Omega) \ni \sum_{\mathbf{k} \in \mathbb{Z}} \mathbf{v}_{\mathbf{k}} e^{i \mathbf{k} \cdot \mathbf{x}} \longmapsto \sum_{|\mathbf{k}|_{\infty} \leq N} \mathbf{v}_{\mathbf{k}} e^{i \mathbf{k} \cdot \mathbf{x}} \in \mathbb{P}_{N}$ be the usual truncation operator. All fields $\mathbf{v}$ can be decomposed as follows: $\mathbf{v}=P_{\varepsilon} \mathbf{v}+$ $\left(1-P_{\varepsilon}\right) \mathbf{v}$. The component $P_{\varepsilon} \mathbf{v}$ in $\mathbf{X}_{\varepsilon}$ is called the large scale component of $\mathbf{v}$ and the remainder $\left(1-P_{\varepsilon}\right) \mathbf{v}$ is called the small scale component.

The nonlinear Galerkin method can be recast into the following form: Seek $\mathbf{u}_{\varepsilon}$ and $p_{\varepsilon}$ in the Leray class such that

$$
\left\{\begin{array}{l}
\partial_{t} P_{\varepsilon} \mathbf{u}_{\varepsilon}-\nu \nabla^{2} \mathbf{u}_{\varepsilon}+P_{\varepsilon} \mathbf{u}_{\varepsilon} \cdot \nabla \mathbf{u}_{\varepsilon}+\nabla p_{\varepsilon},=\mathbf{f} \\
\nabla \cdot \mathbf{u}_{\varepsilon}=0,\left.\quad P_{\varepsilon} \mathbf{u}_{\varepsilon}\right|_{t=0}=P_{\varepsilon} \mathbf{u}_{0}
\end{array}\right.
$$

It is then possible to prove that (9) has a unique solution and that this solution converges, up to subsequences, to a suitable weak solution of (1). 


\section{Discretization}

The purpose of this section is to introduce discrete versions of some of the pre-LES-models described above. In each case, we show that the requirement for the approximate solutions to be suitable approximations determines the relationship between the mesh size $h$ and the large eddy scale $\varepsilon$, thus solving a question very often left open or simply heuristically answered in the LES literature.

\subsection{The discrete hyperviscosity model}

We turn our attention to the hyperviscosity model introduced in $\S 3.1$ and we construct a Galerkin-Fourier approximation assuming that $\Omega$ is the torus $(0,2 \pi)^{3}$. Let $N \in \mathbb{N} \backslash\{0\}$ and introduce the meshsize and large eddy scale

$$
h=N^{-1}, \quad \varepsilon=h^{\theta},
$$

where $0<\theta<1$. We set $N_{i}=\frac{1}{\varepsilon}=N^{\theta}$. To approximate the velocity and the pressure fields we introduce the following finite-dimensional vector spaces:

$$
\mathbf{X}_{h}=\dot{\mathbb{P}}_{N}, \quad \text { and } \quad M_{h}=\dot{\mathbb{P}}_{N} .
$$

We introduce $Q(\mathbf{x})=(2 \pi)^{-3} \sum_{N_{i} \leq|\mathbf{k}|_{\infty} \leq N}|\mathbf{k}|^{2 \alpha} e^{i \mathbf{k} \cdot \mathbf{x}}$ where $\alpha>\frac{5}{4}$. The spectral hyperviscosity model consists of the following: Seek $\mathbf{u}_{h} \in \mathcal{C}^{0}\left([0, T] ; \mathbf{X}_{h}\right)$ and $p_{h} \in L^{2}\left([0, T] ; M_{h}\right)$ such that $\forall \mathbf{v} \in \mathbf{X}_{h}, \forall q \in M_{h}$, and a.e. $t$ in $(0, T)$,

$$
\left\{\begin{array}{l}
\left(\partial_{t} \mathbf{u}_{h}, v\right)+\left(\mathbf{u}_{h} \cdot \nabla \mathbf{u}_{h}, v\right)-\left(p_{h}, \nabla \cdot \mathbf{v}\right)+\nu\left(\nabla \mathbf{u}_{h}, \nabla \mathbf{v}\right)+\varepsilon_{N}^{2 \alpha}\left(Q * \mathbf{u}_{h}, \mathbf{v}\right)=(\mathbf{f}, \mathbf{v}), \\
\left(\nabla \cdot \mathbf{u}_{N}, q\right)=0, \forall t \in(0, T],\left.\quad\left(\mathbf{u}_{N}, \mathbf{v}\right)\right|_{t=0}=\left(\mathbf{u}_{0}, \mathbf{v}\right) .
\end{array}\right.
$$

The following result is proved in [5]:

Theorem 1. Let $\mathbf{f} \in L^{2}\left(0, T ; \mathbf{L}^{2}(\Omega)\right)$ and $\mathbf{u}_{0} \in \mathbf{H}^{\alpha}(\Omega) \cap \mathbf{V}$. Assume that $0<\theta<\frac{4 \alpha-5}{4 \alpha}$ if $\alpha \leq \frac{3}{2}$, or $0<\theta<\frac{2(\alpha-1)}{2 \alpha+3}$ otherwise, then the pair $\left(\mathbf{u}_{h}, p_{h}\right)$ is a suitable approximation to (1).

\subsection{The discrete Leray, Leray- $\alpha$, and NLGM models}

Let us keep the same notation as above; in particular, $h=N^{-1}$ and $\varepsilon=h^{\theta}$. Let us approximate $\phi_{\varepsilon} * \mathbf{u}_{\varepsilon}$ in (6) by the truncated Fourier series of $\mathbf{u}_{\varepsilon}$. Then, the discrete Leray model takes the following form: Seek $\mathbf{u}_{h} \in \mathcal{C}^{0}\left([0, T] ; \mathbf{X}_{h}\right)$ and $p_{h} \in L^{2}\left([0, T] ; M_{h}\right)$ such that $\forall \mathbf{v} \in \mathbf{X}_{h}, \forall q \in M_{h}$, and a.e. $t$ in $(0, T)$,

$$
\left\{\begin{array}{l}
\left(\partial_{t} \mathbf{u}_{h}, \mathbf{v}\right)+\left(P_{\varepsilon_{N}} \mathbf{u}_{h} \cdot \nabla \mathbf{u}_{h}, \mathbf{v}\right)-\left(p_{h}, \nabla \cdot \mathbf{v}\right)+\nu\left(\nabla \mathbf{u}_{h}, \nabla \mathbf{v}\right)=(\mathbf{f}, \mathbf{v}) \\
\left(\nabla \cdot \mathbf{u}_{h}, q\right)=0,\left.\quad(\mathbf{u}, \mathbf{v})\right|_{t=0}=\left(\mathbf{u}_{0}, \mathbf{v}\right)
\end{array}\right.
$$


Using again the Fourier setting, the discrete version of the Leray- $\alpha$ model (7) takes the following form: Seek $\mathbf{u}_{h} \in \mathcal{C}^{0}\left([0, T] ; \mathbf{X}_{h}\right)$ and $p_{h} \in L^{2}\left([0, T] ; M_{h}\right)$ such that for all $\mathbf{v} \in \mathbf{X}_{h}$, for all $q \in M_{h}$, and a.e. $t$ in $(0, T)$,

$$
\left\{\begin{array}{l}
\left(\partial_{t} \mathbf{u}_{N}, \mathbf{v}\right)+\left(\overline{\mathbf{u}}_{h} \cdot \nabla \mathbf{u}_{h}, \mathbf{v}\right)-\left(p_{h}, \nabla \cdot \mathbf{v}\right)+\nu\left(\nabla \mathbf{u}_{h}, \nabla \mathbf{v}\right)=(\mathbf{f}, \mathbf{v}) \\
\left(\overline{\mathbf{u}}_{h}, \mathbf{v}\right)+\varepsilon^{2}\left(\nabla \overline{\mathbf{u}}_{h}, \nabla \mathbf{v}\right)=\left(\mathbf{u}_{h}, \mathbf{v}\right) \\
\left(\nabla \cdot \mathbf{u}_{h}, q\right)=0,\left.\quad\left(\mathbf{u}_{h}, \mathbf{v}\right)\right|_{t=0}=\left(\mathbf{u}_{0}, \mathbf{v}\right)
\end{array}\right.
$$

Still retaining the Fourier setting, the discrete version of NLGM (9) is as follows: Seek $\mathbf{u}_{h} \in \mathcal{C}^{0}\left([0, T] ; \mathbf{X}_{h}\right)$, and $p_{h} \in L^{2}\left(0, T ; M_{h}\right)$ such that $\forall t \in(0, T]$, $\forall \mathbf{v} \in \mathbf{X}_{h}, \forall q \in M_{h}$, and a.e. $t$ in $(0, T)$,

$$
\left\{\begin{array}{l}
\left(\partial_{t} P_{\varepsilon} \mathbf{u}_{h}, \mathbf{v}\right)+\nu\left(\nabla \mathbf{u}_{h}, \nabla \mathbf{v}\right)+\left(P_{\varepsilon} \mathbf{u}_{h} \cdot \nabla \mathbf{u}_{h}, \mathbf{v}\right)-\left(p_{h}, \nabla \cdot \mathbf{v}\right)=(\mathbf{f}, \mathbf{v}), \\
\left(\nabla \cdot \mathbf{u}_{h}, q\right)=0,\left.\quad \mathbf{u}_{h}\right|_{t=0}=P_{\varepsilon} \mathbf{u}_{0} .
\end{array}\right.
$$

The following result holds for the three above approximation techniques:

Theorem 2. Let $\mathbf{f} \in L^{2}\left(0, T ; \mathbf{L}^{2}(\Omega)\right)$ and $\mathbf{u}_{0} \in \mathbf{H}$. If $0<\theta<\frac{2}{3}$, the pair $\left(\mathbf{u}_{h}, p_{h}\right)$ is a suitable approximation to (1).

\subsection{The case of DNS}

A natural question that comes to mind is whether a sequence of Direct Numerical Solutions (DNS) is a suitable approximation. To clarify this issue, let $\mathbf{X}_{h} \subset \mathbf{X}$ and $M_{h} \subset L^{2}(\Omega)$ be two finite-dimensional vector spaces and consider the following Galerkin approximation: Seek $\mathbf{u}_{h} \in \mathcal{C}^{0}\left([0, T] ; \mathbf{X}_{h}\right)$ and $p_{h} \in L^{2}\left([0, T] ; M_{h}\right)$ such that for all $\mathbf{v}_{h} \in \mathbf{X}_{h}$, all $q_{h} \in M_{h}$, and a.e. $t \in(0, T)$

$$
\left\{\begin{array}{l}
\left(\partial_{t} \mathbf{u}_{h}, \mathbf{v}\right)+b_{h}\left(\mathbf{u}_{h}, \mathbf{u}_{h}, \mathbf{v}\right)-\left(p_{h}, \nabla \cdot \mathbf{v}\right)+\nu\left(\nabla \mathbf{u}_{h}, \nabla \mathbf{v}\right)=(\mathbf{f}, \mathbf{v}), \\
\left(q, \nabla \cdot \mathbf{u}_{h}\right)=0, \quad \text { and } \quad\left(\left.\mathbf{u}_{h}\right|_{t=0}, \mathbf{v}\right)=\left(\mathbf{u}_{0}, \mathbf{v}\right)
\end{array}\right.
$$

where $b_{h}$ accounts for the nonlinear term and can be written as follows:

$$
b_{h}(\mathbf{u}, \mathbf{v}, \mathbf{w})=\left\{\begin{array}{l}
\left(\mathbf{u} \cdot \nabla \mathbf{v}+\frac{1}{2} \mathbf{v} \nabla \cdot \mathbf{u}, \mathbf{w}\right), \quad \text { or } \\
\left((\nabla \times \mathbf{u}) \times \mathbf{v}+\frac{1}{2} \nabla\left(\mathcal{K}_{h}(\mathbf{u} \cdot \mathbf{v})\right), \mathbf{w}\right),
\end{array}\right.
$$

where $\mathcal{K}_{h}: L^{2}(\Omega) \longrightarrow M_{h}$ is a linear $L^{2}$-stable interpolation operator.

Owing to standard a priori estimates uniform in $h$, it is clear that the pair $\left(\mathbf{u}_{h}, p_{h}\right)$ complies with items (i) and (ii) of Definition 2. Although it is not known in general whether such a construction yields a suitable solution at the limit, it has been proved in [4] that it is indeed the case when low-order finite elements are used and periodic boundary conditions are enforced. More specifically, let $\pi_{h}: \mathbf{L}^{2}(\Omega) \longrightarrow \mathbf{X}_{h}$ be the $L^{2}$-projection onto $\mathbf{X}_{h}$. We assume that there exists $c>0$ independent of $h$ such that

$$
\forall q_{h} \in M_{h}, \quad\left\|\nabla q_{h}\right\|_{L^{2}} \leq c\left\|\pi_{h} \nabla q_{h}\right\|_{L^{2}} .
$$


This hypothesis is shown to hold in, at least, the following two situations (1) $\mathbf{X}_{h}$ is composed of $\mathbb{P}_{1}$-Bubble $H^{1}$-conforming finite elements and $M_{h}$ is composed of $\mathbb{P}_{1} H^{1}$-conforming finite elements; (2) $\mathbf{X}_{h}$ is composed of $\mathbb{P}_{2}$ $H^{1}$-conforming finite elements, $M_{h}$ is composed of $\mathbb{P}_{1} H^{1}$-conforming finite elements, and no tetrahedron has more than 3 edges on $\partial \Omega$.

Definition 3. We say that $\mathbf{X}_{h}$ (resp. $M_{h}$ ) has the discrete commutator property if there exists $\mathcal{I}_{h} \in \mathcal{L}\left(\mathbf{H}_{\#}^{1}(\Omega) ; \mathbf{X}_{h}\right)$ (resp. $\mathcal{J}_{h} \in \mathcal{L}\left(L^{2}(\Omega) ; M_{h}\right)$ ) such that $\forall \phi$ in $W_{\#}^{2, \infty}(\Omega)$ (resp. $\forall \phi$ in $\left.W_{\#}^{1, \infty}(\Omega)\right)$ and $\forall v_{h} \in \mathbf{X}_{h}$ (resp. $\forall q_{h} \in M_{h}$ )

$$
\begin{aligned}
& \left\|\phi v_{h}-\mathcal{I}_{h}\left(\phi v_{h}\right)\right\|_{H^{l}} \leq c h^{1+m-l}\left\|v_{h}\right\|_{H^{m}}\|\phi\|_{W^{m+1, \infty}}, \quad 0 \leq l \leq m \leq 1 \\
& \left\|\phi q_{h}-\mathcal{J}_{h}\left(\phi q_{h}\right)\right\|_{L^{2}} \leq c h\left\|q_{h}\right\|_{L^{2}}\|\phi\|_{W^{1, \infty}} .
\end{aligned}
$$

Standard $H^{1}$-conforming finite element spaces actually possess the discrete commutator property. This is not the case of Fourier-based approximation spaces since Fourier series do not have local interpolation properties.

The main result is the following (see [4] for details)

Theorem 3. Under the above hypotheses, if $\mathbf{X}_{h}$ and $M_{h}$ have the discrete commutator property, the pair $\left(\mathbf{u}_{h}, p_{h}\right)$ is a suitable approximation to (1).

This result underlines that the nature of the approximation technique that is used plays a key role in the construction of suitable approximations. Low-order approximations seem to do the trick without requiring extra regularization provided the nonlinear term is written in skew-symmetric form, whereas spectral methods need smoothing or extra viscosities. This is related to the fact that spectral methods suffer from the Gibbs phenomenon. This result tends to confirm statements sometimes made in the literature that, when using low-order methods, it is preferable to let the "numerical diffusion do the job" than to perform any LES modeling. This result is also a cautionary notice to LES practitioners that heuristic arguments in the Fourier space may not be equivalent to arguments in the physical space. This point is important since a lot of heuristic LES argumentation is done in the Fourier space.

\section{References}

1. L. Caffarelli, R. Kohn, and L. Nirenberg. Partial regularity of suitable weak solutions of the Navier-Stokes equations. Comm. Pure Appl. Math., 35(6):771831, 1982.

2. A. Cheskidov, D.D. Holm, E. Olson, and E.S. Titi. On a Leray- $\alpha$ model of turbulence. Royal Society London, Proceedings, Series A, Mathematical, Physical \& Engineering Sciences, 461:629-649, 2005.

3. C. Foias, O. Manley, and R. Temam. Modelling of the interaction of small and large eddies in two-dimensional turbulent flows. RAIRO $M^{2} A N, 22: 93-114,1988$.

4. J.-L. Guermond. Finite-element-based Faedo-Galerkin weak solutions to the Navier-Stokes equations in the three-dimensional torus are suitable. J. Math. Pures Appl., 2005, In press. 
5. J.-L. Guermond and S. Prudhomme. Mathematical analysis of a spectral hyperviscosity LES model for the simulation of turbulent flows. Math. Model. Numer. Anal. (M2AN), 37(6):893-908, 2003.

6. Cheng He. On partial regularity for weak solutions to the Navier-Stokes equations. J. Funct. Anal., 211(1):153-162, 2004.

7. A. Leonard. Energy cascade in Large-Eddy simulations of turbulent fluid flows. Adv. Geophys., 18:237-248, 1974.

8. J. Leray. Essai sur le mouvement d'un fluide visqueux emplissant l'espace. Acta Math., 63:193-248, 1934.

9. J.-L. Lions. Sur certaines équations paraboliques non linéaires. Bull. Soc. Math. France, 93:155-175, 1965.

10. V. Scheffer. Hausdorff measure and the Navier-Stokes equations. Comm. Math. Phys., 55(2):97-112, 1977. 